\title{
An Evolutionary Approach to Allocate Frequency in Cellular Telephone System
}

\author{
Anand Kumar \\ Department of Master of Computer Applications \\ AMC Engineering College, Bangalore
}

\author{
Dr. N. N. Jani \\ Department of Computer Science \\ Kadi Sarva Vishwavidyalya. Gandhinagar
}

\begin{abstract}
This paper presents an evolutionary approach (genetic algorithm) to allocate frequencies in the cells of cellular network. In cellular telephone system, each cellular area is divided into small regions called cells. Each cell uses a unique set of frequencies. There is limited frequency so the frequency needs to be reuse. The Frequency allocation problem states that given any area separated into cells are allocated frequencies in such a way that no neighbor cells could have the same frequency... Since the problem looks very simple but as the number of cells is increased it becomes very complex and becomes NP-Complete problem. To find the solution of this problem, we have explored the use of genetic algorithm where possible solutions are improved generation by generation and there is more probability to find the exact solution. . Fitness function is developed which is the backbone of the concept of genetic algorithm and directly affects the performance; since this is NP problem and traditional heuristics have had only limited success in solving small to mid size problems. In this paper we have tried to show that genetic algorithm is an alternative solution for this NP problem where conventional deterministic methods are not able to provide the optimal solution.
\end{abstract}

\section{Keywords}

Genetic Algorithm, Cellular Telephone, Frequency allocation

\section{INTRODUCTION}

In cellular telephone system, each cellular area is divided into small regions called cells. Each cell contains an antenna and is controlled by solar or AC powered network called base station. The size of the cell is not fixed and can be increased or decreased depending on the population of the area. High density area requires more, geographically smaller cells to meet traffic demands than do low-density areas. The transmission of signals is based on is based on Frequency-Reuse Principle [1]. Neighboring cells cannot use the same frequency for communication because it may create interference for the users located near the cell boundaries. However, the set of frequencies available is limited, and frequencies need to be reuse. A frequency reuse pattern is a configuration of $\mathrm{N}$ cells, $\mathrm{N}$ being the reuse factor, in which each cell uses a unique set of frequencies. When the pattern is repeated, the frequencies can be reused. In this paper frequency is reused but the allocation method is not based on the pattern. In frequency pattern method, all the time same pattern is used. If any cell of the pattern need some specific frequency and if it is allotted, then the same pattern with specific frequency will be allotted further, which may not be needed. This is one of the limitations of pattern based allocation method. In this paper method is proposed to eliminate this limitation. The proposed method is based on genetic algorithm. It is also tried to show that only 4 frequencies are capable to allocate any number of cells. The Frequency allocation problem states that given any area separated into cells are allocated frequencies in such a way that no neighbor cells could have the same frequency. Two cells are called adjacent only if they share a border segment. It is an application of Graph coloring problem [2] and graph coloring problem is one of the hardest problems in the class NP (non-deterministic polynomial problems).

\section{A. Cellular Telephony frequency Allocation}

In cellular telephone network entire area is divided into small cells. A cellular area is considered as a Graph. An assignment of frequencies to a cellular area (graph) $\mathrm{G}=(\mathrm{V}, \mathrm{E})$ where $\mathrm{V}$ represents Cells (nodes) and $\mathrm{E}$ represents connectivity (edges) an allocation: $\mathrm{V} \rightarrow \mathrm{F}$, where $\mathrm{F}$ is a finite set of frequencies, the allocation of frequencies is such that if $(v, w) € E$ then $f(v) \neq f$ $(w) ;[5]$ in other words, the same frequency is not assigned to adjacent vertices.

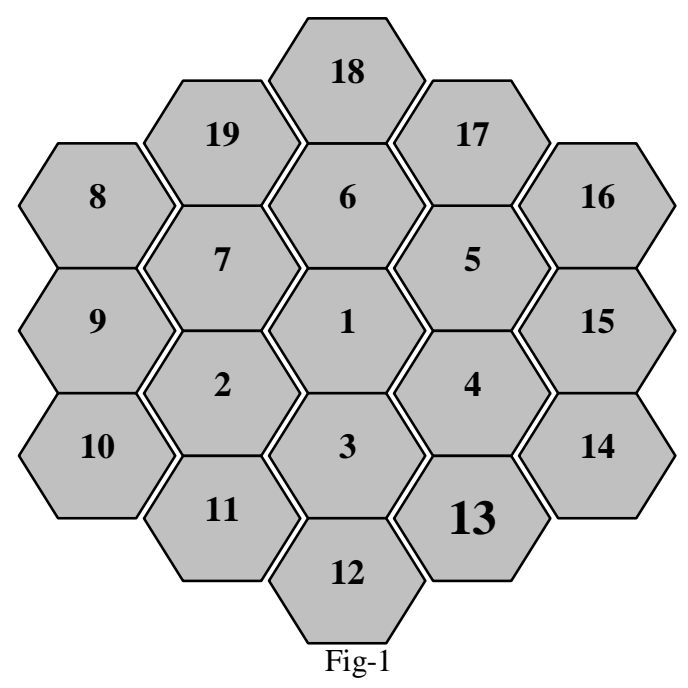

\section{B. Genetic Algorithm}

Genetic algorithms (GA) is a powerful, robust search and optimization tool, which work on the natural concept of evolution, based on natural genetics and natural selection.. Its working principle is completely different, compared to classical search and optimization methods. Because of its broad applicability, ease of use, and global perspective, GAs have been increasingly applied to various search and optimization problems in the recent past. Genetic algorithms are typically implemented as a computer simulation in which a population of abstract representations (called chromosomes) of candidate solutions (called individuals) to an optimization problem evolves toward 
better solutions. Traditionally, solutions are represented in binary as strings of $0 \mathrm{~s}$ and $1 \mathrm{~s}$, but different encoding are also possible [3]. The evolution starts from a population of completely random individuals and happens in generations. This process is repeated generation by generation until the required result is not found. Most importantly of all, GAs are critically dependent on the fitness function. If we can't define the fitness function, then we can't apply a GA.

\section{Work flow of Genetic Algorithm [4]}

1. Initialization of parent population

2. Evaluation

3. Selection

4. Crossover/recombination

5. Mutation

6. Evaluate child and Go to step 3 until termination criteria satisfies

- Initialization of parent population means to generate the $M$ number of solution string known as parent population which is mostly random

- Evaluation means give fitness to each of the solution. It is very important part of the algorithm based on the nature of problem and the requirement of the solution. It varies problem to problem.

- Selection means to select some of the best fit chromosomes from parent population according to some selection criteria (eg. Roulette wheel selection). Fit solution are likely to survive and bad solution are likely to die off

- Crossover/Recombination means to exchange partial solution between pair of selected solution with some probability

- Mutation means change the value of an allele of solution with some small probability value, it is a motivation to explore new point in the solution space

\section{FREQUENCY ALLOCATION PROBLEM PRESENTATION}

The frequency allocation problem in cellular telephone system is represented with the help of an undirected graph where each cell is represented as a vertex of the graph and the connectivity (neighborhood) of the cell is represented as the edge between to vertex. We have considered a cellular area which is divided into 19 cells, marked with numbers $1,2,3 \ldots . .19$. This 19 cells cellular area is considered as a 19 vertex undirected graph which is represented with the help of adjacency matrix. In this matrix representation, each (1) represents adjacency cells. For the cell 1 (vertx-1), its adjacent cells are 2, 3, 4, 5, 6 and 7 .

$$
\text { TABLE -1 }
$$

(a) ADJACENCY MATRIX OF THE GRAPH

\begin{tabular}{|l|l|l|l|l|l|l|l|l|l|l|}
\hline vertex & $\mathbf{1}$ & $\mathbf{2}$ & $\mathbf{3}$ & $\mathbf{4}$ & $\mathbf{5}$ & $\mathbf{6}$ & $\mathbf{7}$ & $\mathbf{8}$ & $\mathbf{9}$ & $\mathbf{1 0}$ \\
\hline $\mathbf{1}$ & 0 & 1 & 1 & 1 & 1 & 1 & 1 & 0 & 0 & 0 \\
\hline $\mathbf{2}$ & 1 & 0 & 1 & 0 & 0 & 0 & 1 & 0 & 1 & 1 \\
\hline $\mathbf{3}$ & 1 & 1 & 0 & 1 & 0 & 0 & 0 & 0 & 0 & 0 \\
\hline $\mathbf{4}$ & 1 & 0 & 1 & 0 & 1 & 0 & 0 & 0 & 0 & 0 \\
\hline $\mathbf{5}$ & 1 & 0 & 0 & 1 & 0 & 1 & 0 & 0 & 0 & 0 \\
\hline $\mathbf{6}$ & 1 & 0 & 0 & 0 & 1 & 0 & 1 & 0 & 0 & 0 \\
\hline $\mathbf{7}$ & 1 & 1 & 0 & 0 & 0 & 1 & 0 & 1 & 1 & 0 \\
\hline $\mathbf{8}$ & 0 & 0 & 0 & 0 & 0 & 0 & 1 & 0 & 1 & 0 \\
\hline $\mathbf{9}$ & 0 & 1 & 0 & 0 & 0 & 0 & 1 & 1 & 0 & 1 \\
\hline $\mathbf{1 0}$ & 0 & 1 & 0 & 0 & 0 & 0 & 0 & 0 & 1 & 0 \\
\hline
\end{tabular}

\begin{tabular}{|l|l|l|l|l|l|l|l|l|l|l|}
\hline $\mathbf{1 1}$ & 0 & 1 & 1 & 0 & 0 & 0 & 0 & 0 & 0 & 1 \\
\hline $\mathbf{1 2}$ & 0 & 0 & 1 & 0 & 0 & 0 & 0 & 0 & 0 & 0 \\
\hline $\mathbf{1 3}$ & 0 & 0 & 1 & 1 & 0 & 0 & 0 & 0 & 0 & 0 \\
\hline $\mathbf{1 4}$ & 0 & 0 & 0 & 1 & 0 & 0 & 0 & 0 & 0 & 0 \\
\hline $\mathbf{1 5}$ & 0 & 0 & 0 & 1 & 1 & 0 & 0 & 0 & 0 & 0 \\
\hline $\mathbf{1 6}$ & 0 & 0 & 0 & 0 & 1 & 0 & 0 & 0 & 0 & 0 \\
\hline $\mathbf{1 7}$ & 0 & 0 & 0 & 0 & 1 & 1 & 0 & 0 & 0 & 0 \\
\hline $\mathbf{1 8}$ & 0 & 0 & 0 & 0 & 0 & 1 & 0 & 0 & 0 & 0 \\
\hline $\mathbf{1 9}$ & 0 & 0 & 0 & 0 & 0 & 1 & 1 & 1 & 0 & 0 \\
\hline
\end{tabular}

\begin{tabular}{|l|l|l|l|l|l|l|l|l|l|}
\hline vertex & $\mathbf{1 1}$ & $\mathbf{1 2}$ & $\mathbf{1 3}$ & $\mathbf{1 4}$ & $\mathbf{1 5}$ & $\mathbf{1 6}$ & $\mathbf{1 7}$ & $\mathbf{1 8}$ & $\mathbf{1 9}$ \\
\hline $\mathbf{1}$ & 0 & 0 & 0 & 0 & 0 & 0 & 0 & 0 & 0 \\
\hline $\mathbf{2}$ & 1 & 0 & 0 & 0 & 0 & 0 & 0 & 0 & 0 \\
\hline $\mathbf{3}$ & 1 & 1 & 1 & 0 & 0 & 0 & 0 & 0 & 0 \\
\hline $\mathbf{4}$ & 0 & 0 & 1 & 1 & 1 & 0 & 0 & 0 & 0 \\
\hline $\mathbf{5}$ & 0 & 0 & 0 & 0 & 1 & 1 & 1 & 0 & 0 \\
\hline $\mathbf{6}$ & 0 & 0 & 0 & 0 & 0 & 0 & 1 & 1 & 1 \\
\hline $\mathbf{7}$ & 0 & 0 & 0 & 0 & 0 & 0 & 0 & 0 & 1 \\
\hline $\mathbf{8}$ & 0 & 0 & 0 & 0 & 0 & 0 & 0 & 0 & 1 \\
\hline $\mathbf{9}$ & 0 & 0 & 0 & 0 & 0 & 0 & 0 & 0 & 0 \\
\hline $\mathbf{1 0}$ & 1 & 0 & 0 & 0 & 0 & 0 & 0 & 0 & 0 \\
\hline $\mathbf{1 1}$ & 0 & 1 & 0 & 0 & 0 & 0 & 0 & 0 & 0 \\
\hline $\mathbf{1 2}$ & 1 & 0 & 1 & 0 & 0 & 0 & 0 & 0 & 0 \\
\hline $\mathbf{1 3}$ & 0 & 1 & 0 & 1 & 0 & 0 & 0 & 0 & 0 \\
\hline $\mathbf{1 4}$ & 0 & 0 & 1 & 0 & 1 & 0 & 0 & 0 & 0 \\
\hline $\mathbf{1 5}$ & 0 & 0 & 0 & 1 & 0 & 1 & 0 & 0 & 0 \\
\hline $\mathbf{1 6}$ & 0 & 0 & 0 & 0 & 1 & 0 & 1 & 0 & 0 \\
\hline $\mathbf{1 7}$ & 0 & 0 & 0 & 0 & 0 & 1 & 0 & 1 & 0 \\
\hline $\mathbf{1 8}$ & 0 & 0 & 0 & 0 & 0 & 0 & 1 & 0 & 1 \\
\hline $\mathbf{1 9}$ & 0 & 0 & 0 & 0 & 0 & 0 & 0 & 1 & 0 \\
\hline
\end{tabular}

\section{GENETIC ALGORITHM APPROACH TO SOLVE THE PROBLEM}

\author{
Simple Genetic Algorithm \\ \{ \\ Initialize population; \\ Evaluate population \\ While Termination-Criteria-Not-Satisfied \\ Select parents for reproduction; \\ Perform crossover/recombination \\ Evaluate population; \\ Perform mutation; \\ Evaluate population;$$
\text { \} }
$$ \\ \}
}

The simple genetic algorithm approach is slightly changed by applying the evaluation function after the use of each genetic operator because of its uncertain nature.

\section{INITIALIZATION OF PARENT POPULATION}

Here 4 sets of chromosomes a, b, c and d are generated randomly with the help of a function. It is called parent population. Each chromosome is represented as a sequence of digits. There are 
only 4 digits which represents four different frequencies. We have tried to show that 4 different frequencies are sufficient to allocate any number of regions which is also a chromatic number.

Four different frequencies are: 1-f1, 2-f2, 3-f3 and 4-f4

Randomly generated chromosomes are.

\begin{tabular}{|l|l|l|l|l|l|l|l|l|l|l|l|l|l|l|l|l|l|l|l|}
$\mathbf{a}$ & 1 & 3 & 2 & 4 & 3 & 2 & 4 & 1 & 2 & 1 & 2 & 1 & 2 & 3 & 4 & 2 & 4 & 3 & 2 \\
\hline
\end{tabular}

\begin{tabular}{|l|l|l|l|l|l|l|l|l|l|l|l|l|l|l|l|l|l|l|l|}
\hline $\mathbf{b}$ & 3 & 2 & 4 & 1 & 3 & 2 & 4 & 1 & 4 & 3 & 4 & 1 & 3 & 1 & 2 & 1 & 4 & 1 & 3 \\
\hline
\end{tabular}

\begin{tabular}{|l|l|l|l|l|l|l|l|l|l|l|l|l|l|l|l|l|l|l|l|} 
c & 1 & 2 & 3 & 4 & 1 & 2 & 3 & 4 & 3 & 2 & 4 & 1 & 2 & 3 & 4 & 2 & 3 & 2 & 1 \\
\hline
\end{tabular}

\begin{tabular}{|l|l|l|l|l|l|l|l|l|l|l|l|l|l|l|l|l|l|l|l|}
$\mathbf{d}$ & 3 & 4 & 2 & 3 & 4 & 1 & 4 & 1 & 2 & 3 & 1 & 2 & 3 & 1 & 2 & 1 & 2 & 3 & 2 \\
\hline
\end{tabular}

\section{Evaluation}

Evaluation is based on fitness function. All the randomly generated chromosomes are evaluated with the help of fitness functions developed. There are two fitness function developed: first to check the specific frequency in specific cell and the second is to check the, no two contiguous cells could hold the same frequency.

\section{1) Contiguous cell frequency check}

This Fitness function is the backbone of this evolutionary approach which is developed to check that how many cells have different frequencies with its adjacent cells for each set of randomly generated chromosomes. For a particular chromosome and for its particular cell, if there are no same frequencies for any of its contiguous cell, then it earns 1 fitness point.

Let us consider the first randomly generated chromosome a,

This chromosome ' $a$ ' represents the frequency of all the cells sequentially. Each allele of the chromosome represents the frequency of cells from cell no. 1 to cell no. 19 respectively.

From Table -1 , it is found that that, the contiguous cells of cell-1 are cell-2, cell-3, cell-4, cell-5, cell-6 and cell-7. when the allocated frequencies by chromosome-(a) are compared for cell-1 to its contiguous cells, it is found that no contiguous cells of cell1 has the same frequency as the frequency of cell-1. So it will earn fitness point 1 . Similarly when the same method is applied to rest of the cells, the total fitness point earned is 12. This function checks the non-availability of the same frequency in contiguous cells of a particular cell. The contiguous cells for each of the cell are defined in TABLE-1, which is represented by matrix $[\mathrm{N}][\mathrm{N}]$. The randomly generated chromosome is represented with the help of an array chromo [N]
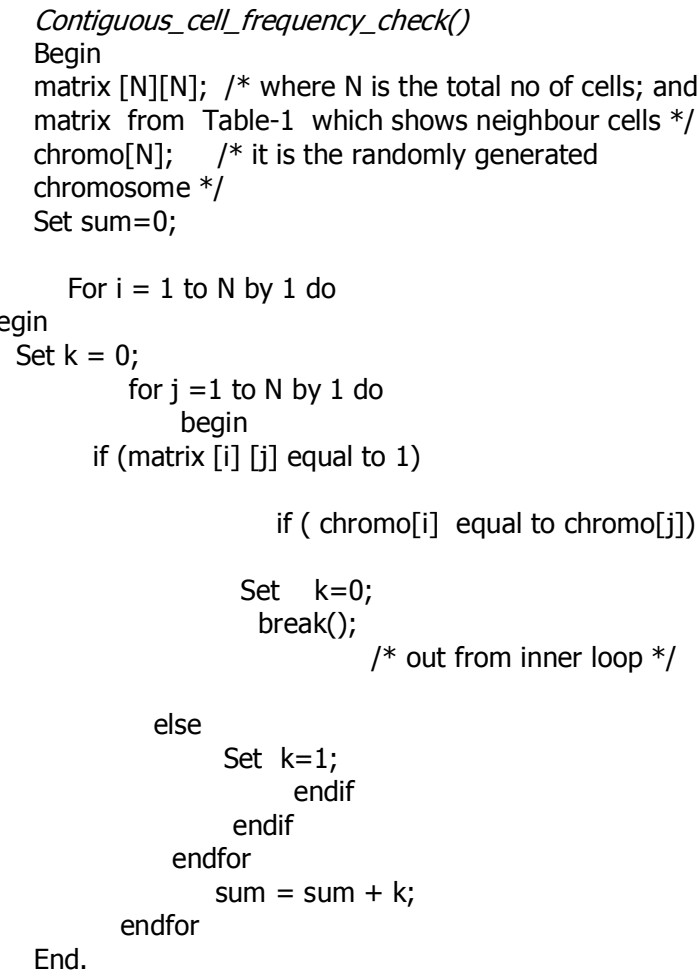

Here sum will return the total fitness point for a randomly generated chromosome.

2) Specific cell frequency check: This function is developed to check the existence of a specific frequency as per demand in the specific cell. In the absence of the required frequency, the chromosome is validated by shifting the same required frequency with the help of mutation operator.

\section{RESULT OF EVALUATION}

When the same function is applied to with all the rest chromosomes b, c, and d we find the following fitness..

TABLE - 2

FITNESS POINT FOR PARENT POPUATION

\begin{tabular}{|c|c|}
\hline Parent population & Fitness \\
\hline a & 12 \\
\hline b & 11 \\
\hline c & 09 \\
\hline d & 12 \\
\hline
\end{tabular}

We know that total no of cells are 19, and that is why maximum fitness should be 19 for any of the chromosome, which is not achieved. It means that any of the chromosomes are not capable to allocate the required frequency. In this condition, according to the genetic algorithm approach, next step is next generation with the use of genetic operators. 


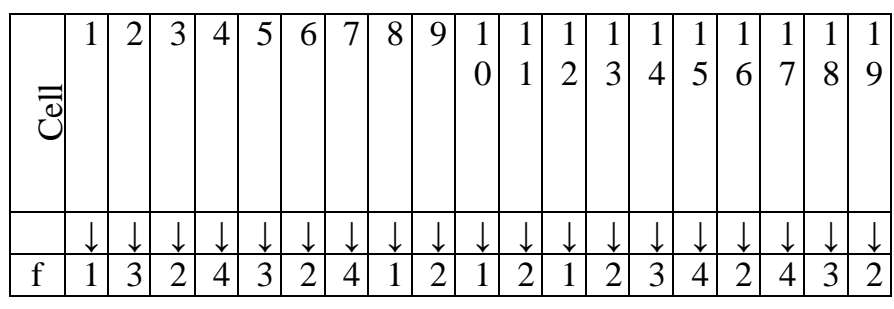

A. Selection

In genetic algorithm fit solution are likely to survive and bad solution are likely to die off. So some of the best fit chromosomes are selected from parent population according to some selection criteria (e.g. Roulette wheel selection). Selected chromosomes are a, b, c, and d.

\section{B. Crossover/Recombination}

Selected solutions are used for crossover. We have considered 1 point cross over.

After crossover of a and $\mathrm{b}$ following child solutions $\mathrm{x}$ and $\mathrm{y}$ are found.

Similarly c and d populations are used for crossover

After crossover of $\mathrm{c}$ and $\mathrm{d}$ following child solutions $\mathrm{p}$ and $\mathrm{q}$ are found

\section{Result of Evaluation}

Due to uncertain nature of genetic algorithm, it is important to evaluate the fitness of chid population because we don't know that when can we found the required result

TABLE -3

\section{FITNESS POINT FOR CHILD POPUATION}

\begin{tabular}{|c|c|}
\hline Child population & Fitness \\
\hline $\mathbf{x}$ & $\mathbf{1 9}$ \\
\hline $\mathbf{y}$ & 11 \\
\hline $\mathbf{p}$ & 07 \\
\hline $\mathbf{q}$ & 06 \\
\hline
\end{tabular}

From the table- 3 , it is clear that the maximum fitness point have been achieved by child population $\mathbf{x}$. If any of the chromosomes does not have the fitness point 19 , then other genetic operator mutation is applied. From the fitness value we found that child population $\mathrm{x}$ has the maximum fitness value 19 which is required. It means that all the nodes (cells) will have different frequencies which was the objective of this study. If the required solution is not found then this child population replaces the parent population and the entire process is restarted from selection phase.

After applying the colours recommended by chromosome $\mathrm{x}$, the resultant map will appear like..

\begin{tabular}{|l|l|l|l|l|l|l|l|l|l|l|l|l|l|l|l|l|l|l|l|}
\hline $\mathbf{a}$ & 1 & 3 & 2 & 4 & 3 & 2 & 4 & 1 & 2 & 1 & 2 & 1 & 2 & 3 & 4 & 2 & 4 & 3 & 2 \\
\hline
\end{tabular}

\begin{tabular}{|l|l|l|l|l|l|l|l|l|l|l|l|l|l|l|l|l|l|l|l|}
\hline $\mathbf{b}$ & 3 & 2 & 4 & 1 & 3 & 2 & 4 & 1 & 4 & 3 & 4 & 1 & 3 & 1 & 2 & 1 & 4 & 1 & 3 \\
\hline
\end{tabular}

\begin{tabular}{|l|l|l|l|l|l|l|l|l|l|l|l|l|l|l|l|l|l|l|l|}
\hline $\mathrm{x}$ & 1 & 3 & 2 & 4 & 3 & 2 & 4 & 1 & 2 & 1 & 4 & 1 & 3 & 1 & 2 & 1 & 4 & 1 & 3 \\
\hline
\end{tabular}

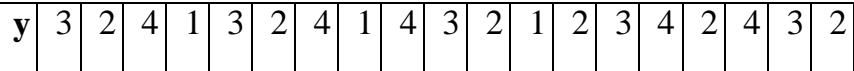

\begin{tabular}{|l|l|l|l|l|l|l|l|l|l|l|l|l|l|l|l|l|l|l|}
\hline $\mathrm{C}$ & 1 & 2 & 3 & 4 & 1 & 2 & 3 & 4 & 3 & 2 & 4 & 1 & 2 & 3 & 4 & 2 & 3 & 2 \\
\hline
\end{tabular}

\begin{tabular}{|l|l|l|l|l|l|l|l|l|l|l|l|l|l|l|l|l|l|l|l|}
\hline $\mathrm{d}$ & 3 & 4 & 2 & 3 & 4 & 1 & 4 & 1 & 2 & 3 & 1 & 2 & 3 & 1 & 2 & 1 & 2 & 3 & 2 \\
\hline
\end{tabular}

\begin{tabular}{|l|l|l|l|l|l|l|l|l|l|l|l|l|l|l|l|l|l|l|l|}
$\mathrm{p}$ & 1 & 2 & 3 & 4 & 1 & 2 & 3 & 4 & 3 & 2 & 1 & 2 & 3 & 1 & 2 & 1 & 2 & 3 & 2 \\
\hline
\end{tabular}

\begin{tabular}{|l|l|l|l|l|l|l|l|l|l|l|l|l|l|l|l|l|l|l|l|}
\hline $\mathrm{q}$ & 3 & 4 & 2 & 3 & 4 & 1 & 4 & 1 & 2 & 3 & 4 & 1 & 2 & 3 & 4 & 2 & 3 & 2 & 1 \\
\hline
\end{tabular}

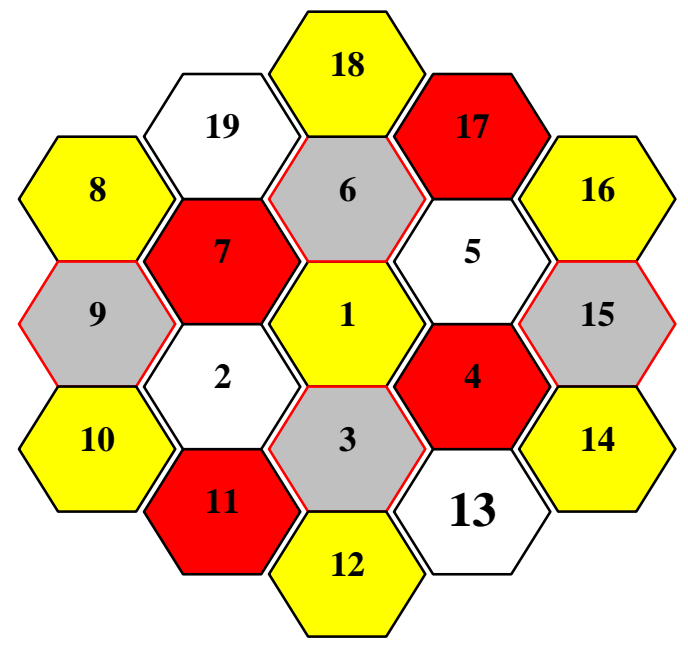

Fig-2

\section{Mutation}

We have explored the new definition of mutation. Only those chromosome will be mutated which does not hold the required frequency in specific cell. The required cell will be mutated with the missing frequency.

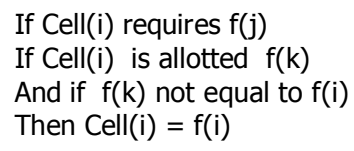

Where Cell(i ) represents specific cell no, $f(j)$ and $f(k)$ represents specific frequency. 


\section{CONCLUSIONS}

In this paper, we have proposed a simple Genetic Algorithm approach with little modification in approach, to allocate frequencies in cellular telephony system. The changed approach is to use evaluation function after each step of genetic operators. This is done because of uncertain nature of Genetic Algorithm. The second change is done in mutation operator. We have described the problem and GA that encodes the candidate solution to the problem in a novel way. Each chromosome is a string of frequencies associated with the cells of considered region. The evolutionary approach of GA has shown to be a robust and efficient alternative for the kind of graph colouring problem. The effectiveness of the methodology however can be increased by applying the various genetic operators and the densely connected regions.

\section{ACKNOWLEDGEMENTS}

Our thanks to the reviewers for their cogent and insightful comments.

\section{REFERENCES}

[1] Behrouz A Forouzan. 2006. Data Communications and Networking. The McGraw- Hill Company. ISBN-13: 978-007-06341-5

[2] David E. Goldberg. 1989. Genetic Algorithms in search, optimisation and machine learning. Addison-Wesley.0-20115767-5

[3] Mitchell, M. 1998. An Introduction to genetic Algorithm. MIT Press. 0-262-113316-4(HB)

[4] Michael D. Vose. 1999. The simple genetic algorithm : (PHI)

[5] Narsingh Deo, 2000. Graph Theory with Applications to Engineering and Computer science: (PHI) 